\title{
A NEW PROOF OF A COMPARISON THEOREM FOR ELLIPTIC EQUATIONS
}

KURT KREITH

The classical Sturmian comparison theorem deals with solutions $u, v$ of two ordinary differential equations.

$$
\begin{aligned}
& \frac{d}{d x}\left(a \frac{d u}{d x}\right)+f u=0, \\
& \frac{d}{d x}\left(b \frac{d v}{d x}\right)+g v=0,
\end{aligned}
$$

where $a$ and $b$ are positive and continuously differentiable on a closed interval $I$ and $f$ and $g$ are continuous on $I$. The theorem states that if $a-b \geqq 0$ and $f \leqq g$ on $I$ and $x_{1}, x_{2}$ are zeros of $u$ in $I$, then $v$ has a zero in $\left[x_{1}, x_{2}\right]$.

This theorem was generalized to self-adjoint second order elliptic partial differential equations by Hartman and Wintner [1]. The purpose of this note is to present a new and simple proof of this generalization.

Let $u$ and $v$ be solutions of the elliptic equations

$$
\begin{array}{ll}
\sum_{i, j=1}^{n} \frac{\partial}{\partial x_{j}}\left(a_{i j} \frac{\partial u}{\partial x_{i}}\right)+f u=0, & a_{i j}=\bar{a}_{j i} \\
\sum_{i, j=1}^{n} \frac{\partial}{\partial x_{j}}\left(b_{i j} \frac{\partial v}{\partial x_{i}}\right)+g v=0, & b_{i j}=\bar{b}_{j i}
\end{array}
$$

in a bounded domain $R \subset E^{n}$. We assume that the $a_{i j}$ and $b_{i j}$ are of class $C^{\prime}$ and that $f$ and $g$ are real and continuous in $\bar{R}$. The ellipticity of (1) and (2) requires that the hermitean matrices $A=\left(a_{i j}\right)$ and $B=\left(b_{i j}\right)$ be positive definite in $\bar{R}$.

Theorem. If $G$ is a bounded domain, $\bar{G} \subset R$, and

(i) $u=0$ on $\partial G$.

(ii) $A-B$ is non-negative definite in $\bar{G} .^{1}$

(iii) $g \geqq f$ in $\bar{G}$.

Then $v$ must have a zero in $\bar{G}$.

Proof. Since $f$ is continuous in $\bar{R}$ we can choose a constant $c$ so

Received by the editors December 12, 1961.

1 This is equivalent to the condition " $B^{-1}-A^{-1}$ is non-negative definite" which is used in [1]. 
that $f(x)+c>0$ in $\bar{R}$. Let $F(x)=f(x)+c$. Define the operator

$$
L=-\frac{1}{F} \sum \frac{\partial}{\partial x_{j}}\left(a_{i j} \frac{\partial}{\partial x_{i}}\right)+\frac{c}{F} .
$$

Equation (1) states that $u$ is an eigenfunction of the boundary problem

$$
\begin{aligned}
L u & =\lambda u, \\
u & =0 \text { on } \partial G
\end{aligned}
$$

corresponding to the eigenvalue $\lambda=1$. Since the nodal lines of $u$ divide $G$ into a finite number of domains in which $u \neq 0$ it is sufficient to prove that the theorem holds for each such domain. In other words we may assume that $u \neq 0$ in $G$ and that $\lambda_{1}=1$.

Suppose $v \neq 0$ in $\bar{G}$. Then we can choose a domain $H$ satisfying $\bar{G} \subset H \subset R$ and $v \neq 0$ in $H$. Define

$$
M=-\frac{1}{F} \sum \frac{\partial}{\partial x_{j}}\left(b_{i j} \frac{\partial}{\partial x_{i}}\right)+1-\frac{g}{F} .
$$

Let $n$ denote the exterior normal to $\partial H$. Then, by an appropriate choice of a function $\sigma$ on $\partial H$, equation (2) states that $v$ is an eigenfunction of the boundary value problem

$$
\begin{aligned}
M v & =v v \\
v+\sigma \frac{\partial v}{\partial n} & =0 \text { on } \partial H
\end{aligned}
$$

corresponding to the eigenvalue $\nu=1$. Since $v \neq 0$ in $H, \nu_{1}=1$. Since $\bar{G} \subset H$, classical variational principles guarantee that the first eigenvalue of the boundary problem

$$
\begin{aligned}
M w & =\mu w, \\
w & =0 \text { on } \partial G
\end{aligned}
$$

will satisfy $\mu_{1}>\nu_{1}=1$. In particular, the assumption $v \neq 0$ in $\bar{G}$ has lead to the conclusion $\mu_{1}>\lambda_{1}$. We shall show that this conclusion is untenable.

Assuming

$$
\int_{G} F|u|^{2} d x=\int_{G} F|w|^{2} d x=1
$$

the minimal property of $\mu_{1}$ yields 


$$
\mu_{1}=\int_{\sigma} F \bar{w} M w d x \leqq \int_{G} F \bar{u} M u d x
$$

Writing

$$
M=-\frac{1}{F} \sum \frac{\partial}{\partial x_{j}}\left(b_{i j} \frac{\partial}{\partial x_{i}}\right)+\frac{c}{F}+\left(1-\frac{g+c}{f+c}\right)
$$

and noting that

$$
\begin{aligned}
1-\frac{g+c}{f+c} & \leqq 0 \text { in } G, \\
\sum b_{i j} \xi_{i} \xi_{j} & \leqq \sum a_{i j} \xi_{i} \bar{\xi}_{j} \quad \text { for all }\left(\xi_{1}, \cdots, \xi_{n}\right)
\end{aligned}
$$

we have

$$
\begin{aligned}
\int_{G} F \bar{u} M u d x & =\int_{G}\left[\sum b_{i j} \frac{\partial u}{\partial x_{i}} \frac{\partial \bar{u}}{\partial x_{j}}+c|u|^{2}+F\left(1-\frac{g+c}{f+c}\right)|u|^{2}\right] d x \\
& \leqq \int_{G}\left[\sum a_{i j} \frac{\partial u}{\partial x_{i}} \frac{\partial \bar{u}}{\partial x_{j}}+c|u|^{2}\right] d x=\lambda_{1} .
\end{aligned}
$$

Thus we conclude that $\mu_{1} \leqq \lambda_{1}$ and that $v(x)=0$ for some $x$ in $\bar{G}$.

\section{BibliogRAPHY}

1. P. Hartman and A. Wintner, On a comparison theorem for self-adjoint partial differential equations of elliptic type, Proc. Amer. Math. Soc. 6 (1955), 862.

University of California, Davis 